\title{
Diagnostic Value of Serum Adenosine Deaminase (ADA) Level for Pulmonary Tuberculosis
}

\author{
Shokrollah Salmanzadeh ${ }^{1}$; Heshmatollah Tavakkol ${ }^{2}$; Khalid Bavieh ${ }^{3}$; Seyed Mohammad \\ Alavi ${ }^{1, *}$ \\ ${ }^{1}$ Health Research Institute ,Infectious and Tropical Disease Research Center, Ahvaz Jundishapur University of Medical Sciences, Ahvaz, Ahvaz, IR Iran \\ ${ }^{2}$ Department of Pulmonology, Ahvaz Jundishapur University of Medical Sciences, Ahvaz, IR Iran \\ 3 Department of Internal Medicine, Imam Khomeini Hospital, Ahvaz Jundishapur University of Medical Sciences, Ahvaz, IR Iran \\ *Corresponding author: Seyed Mohammad Alavi, Health Research Institute ,Infectious and Tropical Disease Research Center, Ahvaz Jundishapur University of Medical Sciences, \\ Ahvaz, Ahvaz, IR Iran. Tel: +98-6133387724, Fax: +98-6133335396, E-mail: alavi.seyedmohammad@yahoo.com \\ Received: July 4, 2014; Revised: September 17, 2014; Accepted: September 30, 2014
}

Background: Diagnosis of tuberculosis (TB) is not always easy, thus employing methods with a short duration and acceptable sensitivity and specificity is necessary to diagnose TB.

Objectives: The aim of this study was to investigate the diagnostic value of serum adenosine deaminase (ADA) level for diagnosis of pulmonary tuberculosis.

Patients and Methods: A total of 160 sex and age-matched subjects were included in this study, and were divided to four groups; forty patients with pulmonary tuberculosis (PTB) diagnosed based on the national TB program (NTP), forty patients with non-tuberculosis bacterial pneumonia, forty patients with lung cancer and forty people who were healthy in every respect. Serum adenosine deaminase activity in patients of each group was measured by the Giusti and Galanti calorimetry method using a commercial kit(Diazyme, USA). The ANOVA analysis was used to compare groups for quantitative variables.

Results: Mean serum ADA level in the PTB group was clearly higher than the mean serum ADA in the other three groups. Mean serum ADA was $26 \mathrm{IU} / \mathrm{L}$ in PTB patients, $19.48 \mathrm{IU} / \mathrm{L}$ in patients with pneumonia, $15.8 \mathrm{IU} / \mathrm{L}$ in patients with lung cancer, and $10.7 \mathrm{IU} / \mathrm{L}$ in the control group $(\mathrm{P}<0.05)$. In regard to the cut off value of $26 \mathrm{IU} / \mathrm{L}$ for ADA in patients with PTB sensitivity and specificity was defined as $35 \%$ and $91 \%$, respectively.

Conclusions: Serum ADA activity with high specificity percentage may be a useful alternative test in restricted resource areas to rule out diagnosis of PTB. However, serum ADA activity is not a useful tool for TB diagnosis.

Keywords: Pulmonary, Tuberculosis; Adenosine Deaminase; Sensitivity and Specificity

\section{Background}

Although tuberculosis (TB) is a known infectious disease with a definite epidemiological pattern and known principles of treatment since 60 years ago, yet there is still a considerable number of TB patients in many parts of the world including our country, who are not timely diagnosed and properly treated $(1,2)$. Tuberculosis remains to be a major health problem in many parts of the world including Iran (3). Approximately a third of the world's populations who are infected with Mycobacterium tuberculosis are at risk of developing TB disease. Each year, about nine million active TB cases occur and 1.5 to 2 million people die of this disease worldwide (2). Although the standard method for TB diagnosis is direct observation of acid-fast bacilli in sputum smear or $M$. tuberculosis isolation in specific culture media (1-3), yet this method is not always easy to perform. Therefore, employing other methods with shorter duration and acceptable sensitivity and specificity is necessary to diagnose TB in special situations (4).

Although PCR is the most sensitive and rapid method for diagnosis of $\mathrm{TB}$, yet due to its high costs, it is not available in limited resource areas (5). For this purpose, activity of adenosine deaminase (ADA) enzyme in serum and body fluids, such as sputum, pleural effusion, ascites and cerebrospinal fluid has been taken into consideration $(1,6,7)$. The ADA is an enzyme (EC 3.5.4.4) that is involved in the metabolism of purine and catalyzes the hydrolytic deamination of adenosine to inosine, and deoxyadenosine to deoxyinosine. This enzyme plays an important physiological role in regulation of the effects of these metabolites on immunological, neurological and vascular processes $(1,6-8)$. The ADA plays an important role in proliferation and differentiation of lymphocytes, particularly $\mathrm{T}$ lymphocytes (9). Increased serum ADA activity can be seen in diseases associated with cellular system stimulation, such as typhoid fever, infectious mononucleosis, liver disease, sarcoidosis, leukemia, brucellosis, acute pneumonia, rheumatoid arthritis, malignancies and tuberculosis $(10,11)$. Although, serum ADA level in pulmonary TB patients is higher than in normal

Copyright (C) 2015, Ahvaz Jundishapur University of Medical Sciences. This is an open-access article distributed under the terms of the Creative Commons Attribution-NonCommercial 4.0 International License (http://creativecommons.org/licenses/by-nc/4.0/) which permits copy and redistribute the material just in noncommercial usages, provided the original work is properly cited. 
individuals, $\mathrm{ADA}$ should not be considered as a suitable marker for differentiating between pulmonary TB and other pulmonary infections (12).

\section{Objectives}

Our region is endemic for tuberculosis and in some areas expert laboratory technicians are not available and in some cases, the patients are unable to give proper sputum samples. Because of controversial results about the role of serum ADA level in the diagnosis of PTB, this study aimed to investigate the diagnostic value of serum ADA level (as an alternative method) for diagnosis of PTB.

\section{Patients and Methods}

In this analytical epidemiological study 160 sex and age-matched (as possible as) subjects were included, and were divided to four groups. The first group consisted of forty patients with PTB diagnosed based on the national TB program (NTP) (8) with at least two positive sputum smears or a positive sputum smear plus clinical or radiographic evidence of pulmonary tuberculosis or a positive sputum smear plus a positive sputum culture of $M$. tuberculosis. The exclusion criteria were: patients with lung cancer who also suffered from PTB or patients with PTB associated with other respiratory infections or immunocompromised cases for any reason (congenital or acquired). The other three groups also included forty age and sex-matched patients. The second group included forty patients with non-tuberculous bacterial pneumonia; the third group included forty patients with lung cancer and the fourth group consisted of forty people who were healthy in every aspect (as controls).

After obtaining the informed consent and the completed questionnaires, $3 \mathrm{~mL}$ of blood was taken from each patient and controls under aseptic conditions, and immediately sent to the laboratory. Serum samples after prompt separation from cells or clots were kept tightly stoppered till examination. Serum adenosine deaminase activity was measured by Giusti and Galanti colorimetric method (11) using the ADA assay kit (Diazyme Kit, USA). Serum ADA activity was expressed in IU/L. Every $1 \mathrm{IU} / \mathrm{L}$ of ADA describes the formation of one micro mole of ammonia produced per minute per liter of serum at $37^{\circ} \mathrm{C}$ according manufacture's protocol; validity and calibration of the kit was done by REF DZ117.Con and REF DZ 117 Cal, respectively.

The ANOVA analysis was used to compare groups for quantitative variables, and sensitivity analysis and Crow Rock chart were used to determine the sensitivity and specificity of serum ADA for PTB diagnosis. Receiver Operating Characteristic (ROC) curves were used to determine a cutoff value for the ADA test. A test value below 4.0 was considered to be normal and above 4.0 to be abnormal. A non-parametric method was used if they were not normal. All calculations were performed by the SPSS software version 15

\section{Results}

A total of 160 subjects were included in this study and were divided to four groups (PTB, lung cancer, community acquired pneumonia and healthy subjects). The PTB group consisted of 27 (67.5\%) males and 13 (32.5\%) females with a mean age of 49 years, the lung cancer group consisted of 19 (47.5\%) males and 21 (52.5\%) females with a mean age of 64 years, the CAP group consisted of 21 $(52.5 \%)$ males and $19(47.5 \%)$ females with a mean age of 53 years, and the healthy group consisted of 21 (52.5\%) males and $19(47.5 \%)$ females with a mean age of 41 years. In the PTB group mean serum ADA was clearly higher than the mean serum ADA in the other three groups. Mean serum ADA was $26 \mathrm{IU} / \mathrm{L}$ in patients with PTB, 19.48 IU/L in patients with pneumonia, $15.8 \mathrm{IU} / \mathrm{L}$ in patients with lungs cancer, and $10.7 \mathrm{IU} / \mathrm{L}$ in the control group. Distribution of patients with positive results according to categorized serum ADA cut off points is shown in Table 1.

One-way ANOVA was performed for analysis of data. Before running tests, normality and homogeneity of data were examined. Initially, normality was examined by using the Kolmogrov-Smirnov (KS) sample test. The results of the control group with $\mathrm{P}=0.746$, $\mathrm{PTB}$ patients with $\mathrm{P}=$ 0.106 , lung cancer patients with $\mathrm{P}=0.119$, and pneumonia patients with $\mathrm{P}=0.062$ indicated that the distribution of data was normal (Table 2). Homogeneity of the data was evaluated by Levene's test, considering the $\mathrm{P}$ value of $<0.001$ obtained from this test, it was determined that the data was not homogeneous (Table 2). A P value of $<$ 0.001 was obtained by the Welch test, which generally.

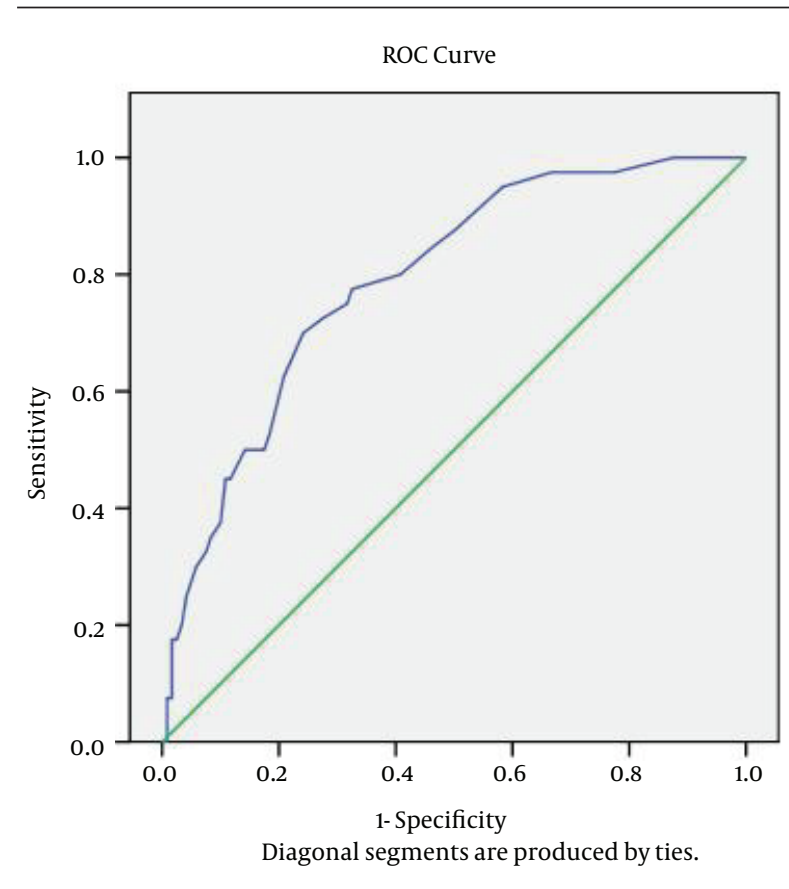

Figure 1. Sensitivity and Specificity of Serum ADA Activity in Studied Subjects 
shows significant differences between the groups (Table 3). Groups were compared two by two using the post hoc test. The test results showed that the TB group was significantly different from the other groups, with $\mathrm{P}<0.001$ compared to the control group, $\mathrm{P}<0.001$ compared to the lung cancer group, and $\mathrm{P}=0.012$ compared to the pneumonia group (Table 3 ).

The sensitivity and specificity of the serum ADA tests were examined by the ROC curve analysis. According to curve coordinates table, if ADA cutoff point is equal to 15.5 , the sensitivity and specificity will be $77 \%$ and $70 \%$, respectively, if ADA cutoff point is equal to 19.5, the sensitivity and specificity will be $62 \%$ and $80 \%$, respectively, and if ADA cut off point is equal to 26, the sensitivity and specificity will be $35 \%$ and $91 \%$, respectively. A higher cut off value, results in higher specificity yet lower sensitivity (Figure 1). In order to test the effect of age on the amount of ADA, nonparametric correlations test was used. To evaluate the effect of gender on the amount of ADA (in regards to older age in cancer patients and higher male proportion in PTB group compared to the other groups), Mann-Whitney test was performed and the results showed that there was no significant relationship between sex and ADA $(P=0.509)$. The results also showed that there was no significant relationship between ADA and age $(\mathrm{P}=0.222)$.

Table 1. Distribution of Patients With Positive Results According to Serum Adenosine Deaminase Cut off Points Among Studied Subjects

\begin{tabular}{|c|c|c|c|c|c|c|c|c|}
\hline \multirow[t]{3}{*}{ Groups } & \multicolumn{6}{|c|}{ Cut Off ${ }^{a}$} & \multirow[t]{3}{*}{ Mean IU/L } & \multirow[t]{3}{*}{ Range IU/L } \\
\hline & \multicolumn{2}{|c|}{$15.5 \mathrm{IU} / \mathrm{L}$} & \multicolumn{2}{|c|}{$19.5 \mathrm{IU} / \mathrm{L}$} & \multicolumn{2}{|c|}{$26 \mathrm{IU} / \mathrm{L}$} & & \\
\hline & Positive & Negative & Positive & Negative & Positive & Negative & & \\
\hline${\text { Pulmonary } \mathrm{TB}^{\mathrm{b}}}^{\mathrm{b}}$ & $31(77.5)$ & $9(22.5)$ & $25(62.5)$ & $15(37.5)$ & $15(37.5)$ & $25(62.5)$ & 26 & $9-59$ \\
\hline Lung cancer & $14(35.0)$ & $26(65.0)$ & $11(27.5)$ & $29(72.5)$ & $5(12.5)$ & $35(87.5)$ & 15.8 & $5-56$ \\
\hline Pneumonia & $26(65.0)$ & $14(35.0)$ & $14(35.0)$ & $26(65.0)$ & $7(17.5)$ & $33(82.5)$ & 19.48 & $3-100$ \\
\hline Healthy subjects & $0(0)$ & $40(100)$ & $0(0)$ & $40(100)$ & $0(0)$ & $40(100)$ & 10.7 & $7-15$ \\
\hline
\end{tabular}

a Data are presented No. (\%).

b $\mathrm{TB}$, tuberculosis.

Table 2. Normality and Homogeneity of Data From the Studied Patients ${ }^{\text {a }}$

\begin{tabular}{lcc}
\hline Groups & Normality of Parameters Sample Kolmogorov-Smirnov & Homogeneity of Parameters Levene's Test \\
\hline Pulmonary tuberculosis & $>0.106$ & $<0.001$ \\
Lung cancer & $>0.119$ & $<0.001$ \\
Pneumonia & $>0.062$ & $<0.001$ \\
Healthy subjects & $>0.746$ & $<0.001$ \\
\hline
\end{tabular}

a Data are presented as P-value.

Table 3. Comparison of Mean Serum Level of Adenosine Deaminase Among Studied Patients a

\begin{tabular}{|lcc}
\hline Variables & Post Hoc Test & Welch Test \\
\hline Pulmonary TB ${ }^{\text {b }}$ & & $<0.001$ \\
\hline Lung cancer & $<0.001$ & $<0.012$ \\
\hline Pneumonia & $<0.001$ & $<0.001$ \\
\hline Healthy Patients & & $<0.001$ \\
\hline Lung Cancer & $<0.001$ & \\
\hline Pulmonary TB & $<0.164$ & $<0.001$ \\
\hline Pneumonia & $<0.048$ & \\
\hline Healthy patients & & \\
\hline Pneumonia & $<0.012$ & \\
\hline Pulmonary TB & $<0.184$ & \\
\hline Lung cancer & $<0.001$ & \\
\hline Healthy patients & & \\
\hline Healthy Subjects & $<0.001$ & \\
\hline Pulmonary TB & $<0.048$ & \\
\hline Lung cancer & $<0.001$ & \\
\hline Pneumonia & & \\
\hline a Data are presented as P-value. & & \\
\hline TB, tuberculosis. & & \\
\hline
\end{tabular}


Salmanzadeh $S$ et al.

\section{Discussion}

Despite all the progress in the diagnosis and treatment of TB, the disease is still a major health problem in many parts of the world, particularly in developing countries. Lack of timely TB diagnosis is the major cause of failure in controlling the disease. Although the standard diagnosis of PTB is based on $M$. tuberculosis isolation or direct observation of AFB in sputum examination yet, other diagnostic methods with shorter duration and acceptable sensitivity and specificity are essential. Rapid diagnosis of PTB from sputum by the Xpert MTB/RIF method using the polymerase chain reaction (PCR) is unavailable in low income countries due to the high costs of this method (5).

In this study, we found that mean serum ADA in patients with pulmonary tuberculosis is clearly higher than in other patients with diseases like pneumonia (26 IU/L vs. 19.5 IU/L, P < 0.012) or lung cancer (26 IU/L vs. 19.5 IU/L, P < $0.001)$. Previous studies have reported that the diagnostic value of serum ADA for pulmonary tuberculosis is associated with controversial results. In studies of Al-shammary from Saudi Arabia (10), Afrasiabian and colleagues from Iran (13) and Dilmac and colleagues from Turkey (14) serum levels of ADA in patients with pulmonary tuberculosis were significantly higher than in other patients with lung cancer and bacterial pneumonia. Agarwal and colleagues in India also showed that serum levels of ADA in patients with sputum smear-negative pulmonary tuberculosis (culture positive) was significantly different from non-tuberculosis patients with other lung diseases such as lung cancer, pneumonia, pulmonary abscess and bronchiectasis (15). Bolursaz et al. believed that although, serum ADA level in pulmonary TB patients is higher than in normal individuals, ADA should not be considered as a suitable marker for differentiating between pulmonary $\mathrm{TB}$ and other pulmonary infections (12).

The current study showed that mean serum ADA in patients with pulmonary TB is significantly higher than in the normal population (26 IU/L vs. $10.7 \mathrm{IU} / \mathrm{L}, \mathrm{P}<0.001$ ). This finding is consistent with studies of Titarenko and colleagues in Russia (16), Agarwalm et al. in India (15), and Afrasiabian and colleagues in Iran (13). Agarwalm and colleagues found that serum levels of ADA in patients with pulmonary TB are significantly higher than in healthy subjects (15). Afrasiabian and colleagues in their study measured serum ADA levels in two groups of patients; cases with pulmonary tuberculosis and patients with non-tuberculosis infections (patients referred to the hospital for surgery) (13). They found that serum levels of ADA in patients with pulmonary TB were significantly higher than in non-tuberculosis subjects (13). In some studies, including a review study by Dinnes et al. significant differences in serum ADA levels, between patients with TB and other patients with non-tuberculosis infection, have not been demonstrated (17).

The current study showed that a serum ADA level of $>$ or
$=26 \mathrm{IU} / \mathrm{L}$ (cut off point) has a sensitivity of $35 \%$ and specificity of $91 \%$ in patients with PTB. When the results of all of our studied patients are compared with the results of patients from other studies, mean serum ADA activities are found to be significantly $(\mathrm{P}<0.05)$ higher in the sera of patients with active PTB (mean, range: 20.8-42.3 IU/L) than in the sera of cancer patients (mean, range: 15.8-20.1 $\mathrm{IU} / \mathrm{L}$ ), community acquired pneumonia patients (mean, range: 15.5-19.5) and healthy controls (mean, range: 5-10.7 $\mathrm{IU} / \mathrm{L})$. For the cut off value range of 15 to $53.5 \mathrm{IU} / \mathrm{L}$ for ADA in patients with PTB the sensitivity and specificity ranged from $12 \%$ to $100 \%$, and $86 \%$ to $100 \%$, respectively. Indeed increase of serum ADA activity cut off point is associated with increased specificity but not sensitivity (except a few studies on children) of the test for diagnosing the active disease of tuberculosis $(1,4,8,14,18-20)$. Our finding related to validity of the serum ADA test is consistent with most previous studies with low sensitivity (range: $12 \%$ - $44 \%$ ) and high (range: $96 \%$ - 100\%) specificity $(1,8,15$, $16)$ yet, disagrees with studies with high (100\%) sensitivity $(18,19)$. Therefore, according to our results indicating low sensitivity for serum ADA level, this test is not a useful tool for TB diagnosis. Based on high specificity for serum ADA level, this test is a useful test to rule out TB in suspected cases with negative microbiological results.

Tarhan and colleagues (21) suggested that serum ADA activities can be used for the diagnosis of tuberculosis as a supplementary laboratory test in combination with clinical and laboratory findings. Our study is similar (with some minor differences) to results obtained from most studies that have been conducted in different parts of the world. These minor differences may be due to accuracy of doing laboratory examination, racial or varied socio-economic status, sample size, site of infection (e.g. pleural), microbiological status (smear positive or smear negative), disease severity, adult versus children and cut off points. To achieve better and more definitive results, further controlled studies are necessary to determine the diagnostic value of ADA activities in patients with active pulmonary tuberculosis.

There were some limitations in our study that have biased our results. This study was the first of its kind in the region to evaluate the capacity of serum ADA in PTB diagnosis therefore conclusions based on one study should be interpreted with caution. Some other diseases such as typhoid fever, infectious mononucleosis, liver disease, sarcoidosis, leukemia, brucellosis and rheumatoid arthritis are expected to be associated with high serum levels of ADA. We have excluded these diseases based on the clinical presentation of PTB, not on the basis of specific diagnostic tests, therefore further controlled studies are recommended. This study showed that serum ADA with high specificity percentage might be a useful alternative test to rule out diagnosis of PTB. Serum ADA activity can be used in the differentiation of non-TB cases from TB cases as a supplementary laboratory test in patients strongly suspected to have negative PTB smear test re- 
sults. However, serum ADA activity is not a useful tool for TB diagnosis. In cases where there is no possibility of using other methods such as sputum culture or polymerase chain technique and in limited source areas with limitation of laboratory staff expert in mycobacteriology, this test could help rule out TB.

\section{Acknowledgements}

This paper was derived from the thesis of Dr Khalid Bavye (no: TB-07) for postgraduate degree of internal medicine (medical ethics approval no: ajums, REC-1392.196). The authors wish to thank the chief and personnel of Jundishapur Infectious and Tropical Research Center for supporting this study as well as the research affair deputy of medical college of Ahvaz Jundishapur University of Medical Sciences for approving the study.

\section{References}

1. Farazi A, Moharamkhani A, Sofian M. Validity of serum adenosine deaminase in diagnosis of tuberculosis. Pan Afr Med J. 2013;15:133.

2. www.who.int/tb. Global Tuberculosis Control, A short update to the 2009 report.: World health organization (WHO) Library Cataloguing-in-Publication Data; 2009. Available from: http://apps.who. int/iris/bitstream/10665/44241/1/9789241598866 eng.pdf?ua=1.

3. Alavi SM, Khoshkhoy MM. Pulmonary tuberculosis and diabetes mellitus: Co-existence of both diseases in patients admitted in a teaching hospital in the southwest of Iran. Caspian J Intern Med. 2012;3(2):421-4.

4. Steingart KR, Ng V, Henry M, Hopewell PC, Ramsay A, Cunningham J, et al. Sputum processing methods to improve the sensitivity of smear microscopy for tuberculosis: a systematic review. Lancet Infect Dis. 2006;6(10):664-74.

5. Jobayer M, Shamsuzzaman SM, Zulfiquer K. Rapid Diagnosis of Pulmonary Tuberculosis From Sputum by Polymerase Chain Reaction. Arch Clin Infect Dis. 2014;9(2)

6. Dimakou K, Hillas G, Bakakos P. Adenosine deaminase activity and its isoenzymes in the sputum of patients with pulmonary tuberculosis. Int J Tuberc Lung Dis. 2009;13(6):744-8.

7. Lamsal M, Gautam N, Bhatta N, Majhi S, Baral N, Bhattacharya SK. Diagnostic utility of adenosine deaminase (ADA) activity in pleural fluid and serum of tuberculous and non-tuberculous respiratory disease patients. Southeast Asian J Trop Med Public Health.
2007;38(2):363-9.

8. Kartaloglu Z, Okutan O, Bozkanat E, Ugan MH, Ilvan A. The course of serum adenosine deaminase levels in patients with pulmonary tuberculosis. Med Sci Monit. 2006;12(11):CR476-80.

9. Russo M, Giancane R, Apice G, Galanti B. Adenosine deaminase and purine nucleoside phosphorylase activities in peripheral lymphocytes from patients with solid tumours. Br J Cancer. 1981;43(2):196-200.

10. al-Shammary FJ. Adenosine deaminase activity in serum and pleural effusions of tuberculous and non-tuberculous patients. Biochem Mol Biol Int. 1997;43(4):763-79.

11. Giusti G, Galanti B. Colorimetric Method. Adenosine deaminase In: Bergmeyer HU, (ed). Methods of enzymatic Analysis. 3rd edWeinheim: Verlag chemie; 1984.

12. Bolursaz MR, Khalilzadeh S, Khodayari A, Hakimi S. Adenosine Deaminase Level as an Indicator for Differentiating Between Active Pulmonary Tuberculosis Infection and Other Pulmonary Infections. J Compr Ped. 2012;3(1):3-6.

13. Afrasiabian S, Mohsenpour B, Bagheri KH, Sigari N, Aftabi K. Diagnostic value of serum adenosine deaminase level in pulmonary tuberculosis. J Res Med Sci. 2013;18(3):252-4.

14. Dilmac A, Ucoluk GO, Ugurman F, Gozu A, Akkalyoncu B, Eryilmaz $\mathrm{T}$, et al. The diagnostic value of adenosine deaminase activity in sputum in pulmonary tuberculosis. Respir Med. 2002;96(8):632-

15. Agarwal MK, Nath J, Mukerji PK, Srivastava VML. A Study of Serum Adenosine Deaminase Activity In Sputum Negative Patients of Pulmonary Tuberculosis. Ind J Tub. 1991;38(1):139-41.

16. Titarenko OT, D'Iakova M E, Perova TL, Riasnianskaia TB. [The activity of adenosine deaminase and its isoenzymes in patients with different forms of pulmonary tuberculosis]. Probl Tuberk. 2002(3):43-5

17. Dinnes J, Deeks J, Kunst H, Gibson A, Cummins E, Waugh N, et al. A systematic review of rapid diagnostic tests for the detection of tuberculosis infection. Health Technol Assess. 2007;11(3):1-196.

18. Kuyucu N, Karakurt C, Bilaloglu E, Karacan C, Tezic T. Adenosine deaminase in childhood pulmonary tuberculosis: diagnostic value in serum. J Trop Pediatr.1999;45(4):245-7.

19. Mishra OP, Yusaf S, Ali Z, Nath G, Das BK. Adenosine deaminase activity and lysozyme levels in children with tuberculosis. JTrop Pediatr. 2000;46(3):175-8.

20. Celik G, Kaya A, Poyraz B, Ciledag A, Elhan AH, Oktem A, et al. Diagnostic value of leptin in tuberculous pleural effusions. Int J Clin Pract. 2006;60(11):1437-42.

21. Tarhan G, Gumuslu F, Yilmaz N, Saka D, Ceyhan I, Cesur S. Serum adenosine deaminase enzyme and plasma platelet factor 4 activities in active pulmonary tuberculosis, HIV-seropositive subjects and cancer patients. J Infect. 2006;52(4):264-8. 